\section{What's UP, DOC?}

\section{Pink eye and fusidic acid}

Is fusidic acid better than placebo for treating acute infectious conjunctivitis? In a randomized controlled trial, I8I adult patients in primary care with (muco)purulent discharge or sticking eyelids were given fusidic gel $1 \%$ or placebo 4 times daily for I week. Recovery rates were the same in the 2 groups, although bacterial eradication was more common in the treatment group (risk difference 35\%, 95\% confidence interval [CI] 9.3\%-60.4\%). Although the study sample is too small to demonstrate equivalence between placebo and fusidic acid, the results suggest that current use of fusidic acid for acute infectious conjunctivitis is unjustified. Br J Gen Pract 2005;55:924-30

\section{Fruit, veg and stroke}

People who eat more than 5 servings of fruit and vegetables per day have $26 \%$ less risk of stroke than those who eat fewer than 3 servings per day (pooled relative risk [RR] 0.76 , 95\% CI $0.69-$ 0.79 ), and eating $3-5$ servings per day reduces the risk by II\% (pooled RR o.89, 95\% CI $0.83-0.97$ ), according to the results of a meta-analysis. Both hemorrhagic and ischemic stroke were reduced. A high consumption of fruit and vegetables (preferably 5 or more servings per day) prevents strokes and should be encouraged. Lancet 2006; 367:320-6

\section{Sibling risk of CVD}

In the Framingham Offspring Study (a cohort of the Framingham Heart Study) people without cardiovascular disease (CVD) were followed for 8 years. CVD in a sibling (occurring before 55 years of age in men and 65 years in women)

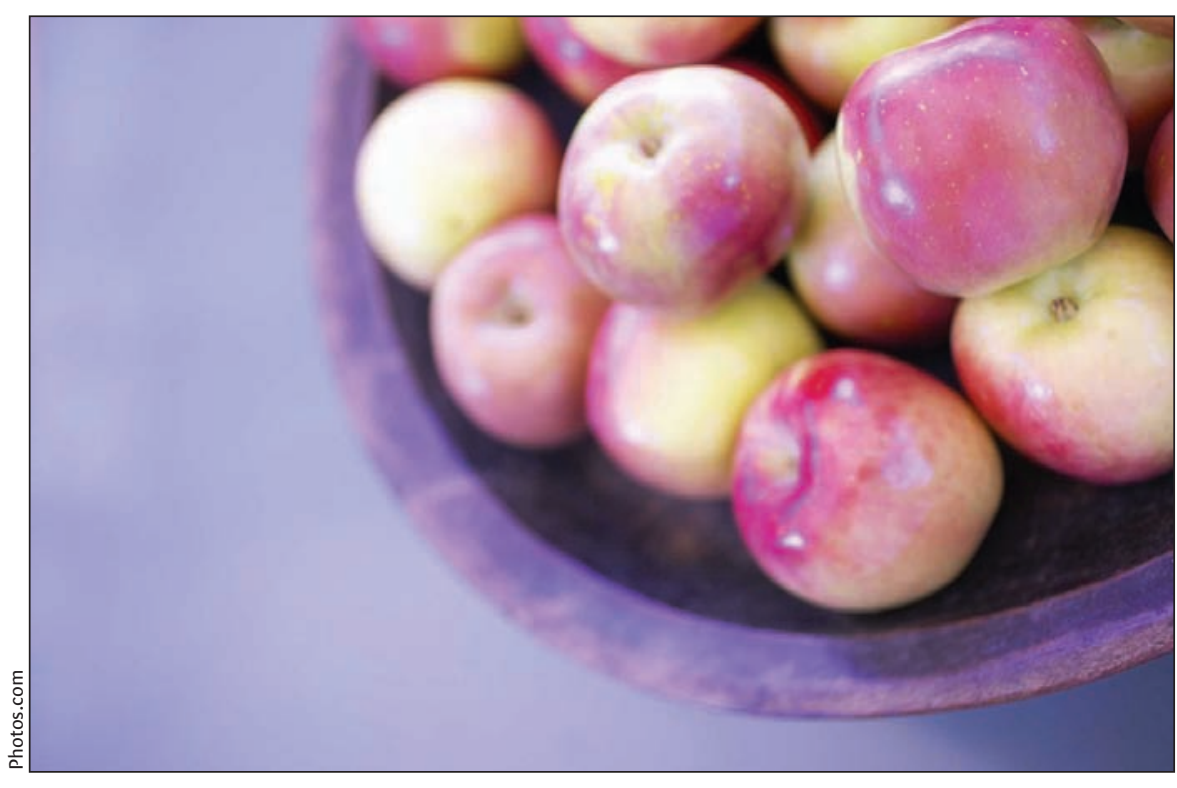

Five apples a day keeps strokes at bay.

was found to increase the personal risk of CVD over and above that associated with early parental CVD, even after adjustment for age, sex, smoking, diabetes and hypertension (odds ratio [OR] I.56, 95\% CI I.II-2.I8). This relation was stronger in people 30-59 years of age (OR 2.22, 95\% CI I.22-4.02). The findings suggest that genetic factors or shared early environmental exposures (in utero or childhood) could play a role in CVD. Sibling CVD should be considered as important as early parental CVD when assessing CVD risk. JAMA 2005;294:3117-23

\section{Intensive diabetes therapy}

\section{and CVD}

For patients with type I diabetes, intensive therapy reduces the risk of microvascular and neurologic complications. Its long-term impact on CVD, however, has been unclear. In the Diabetes Control and Complications Trial, patients were randomly assigned to intensive or conventional therapy for 6.5 years, after which they had the option of intensive or usual therapy (most chose intensive therapy). Intensive therapy consisted of 3 or more daily injections of insulin or treatment with an external insulin pump, with a blood glucose goal of less than Io $\mathrm{mmol} / \mathrm{L}$ after meals and a glycosylated hemoglobin goal of less than $6.05 \%$. After 17 years, researchers looked at long-term development of CVD, defined as stroke, nonfatal myocardial infarction (MI), cardiovascular death, angina or need for revascularization. They found that for patients originally assigned to intensive therapy, the risk of any cardiovascular event was reduced by $42 \%$ (hazard ratio [HR] 0.58, 95\% CI 0.37o.9I) and the risk of nonfatal MI, stroke or cardiovascular death by $57 \%$ (HR $0.43,95 \%$ CI $0.2 \mathrm{I}-0.88$ ), although the absolute difference between the 2 groups was small. Intensive therapy has a long-term beneficial effect on the risk of CVD in patients with type I diabetes. $N$ Engl J Med 2005;353:2643-53 - Compiled by Sally Murray, CMAJ

DOI:I0.1503/cmaj.06oI86 